\title{
Solving Optimal Continuous Thrust Rendezvous Problems with Generating Functions*
}

\author{
Chandeok Park ${ }^{\dagger}$, Daniel J. Scheeres ${ }^{\ddagger}$, and Vincent Guibout ${ }^{\S}$ \\ University of Michigan, Ann Arbor, MI 48109, U.S.A.
}

\begin{abstract}
The optimal control of a spacecraft as it transitions between specified states using continuous thrust in a fixed amount of time is studied using a recently developed technique based on Hamilton-Jacobi theory. Starting from the 1st order necessary conditions for optimality, we derive a Hamiltonian system for the state and adjoints with split boundary conditions. Then, recognizing the two point boundary value problem as a canonical transformation, we employ generating functions to find the optimal feedback control as well as the optimal trajectory. Though we formulate the optimal control problem in the context of the necessary conditions for optimality, our closed-loop solution also formally satisfies the sufficient conditions for optimality via the fundamental connection between the optimal cost function and generating functions. A solution procedure for these generating functions is posed and numerically tested on a non-linear optimal rendezvous problem in the vicinity of a circular orbit. Generating functions are developed as series expansions, and the optimal trajectories obtained from them are compared favorably with those of a numerical solution to the two point boundary value problem using a forward shooting method.
\end{abstract}

\section{Nomenclature}

A linearized system dynamics about the circular reference trajectory

$\arg \min (\cdot) \quad$ argument minimum with respect to the variable $(\cdot)$

$B \quad$ linearized system dynamics about the circular reference trajectory

$\boldsymbol{f}(\boldsymbol{x}, \boldsymbol{u}, t) \quad$ system dynamics

$\boldsymbol{F} \quad$ non-gravitational control force vector

$F_{1}, F_{2}, F_{3}, F_{4}$ principal kinds of generating functions

$H(\boldsymbol{x}, \boldsymbol{p}, \boldsymbol{u}, t)$ Hamiltonian of the system

I identity matrix

$i \quad$ radial unit vector in the rotating coordinate frame

$J \quad$ performance index to be minimized

$j \quad$ tangential unit vector in the rotating coordinate frame

$\boldsymbol{k} \quad$ normal unit vector in the rotating coordinate frame

$L(\boldsymbol{x}, \boldsymbol{u}, t) \quad$ full time performance index or Lagrangian

$m \quad$ mass of the spacecraft, assumed constant in the current application

$p \quad$ adjoint vector

$\boldsymbol{R}=R i \quad$ position vector of the origin of the rotating frame from the central body

$r \quad$ position vector of the spacecraft from the center of gravity

$r$ $|\boldsymbol{r}|$

*Accepted for the Journal of Guidance, Control, and Dynamics, 31 March 2005

${ }^{\dagger} \mathrm{PhD}$. Candidate \& Research Assistant, Department of Aerospace Engineering, FXB 1320 Beal Avenue, Ann Arbor, MI 48109-2140, student member AIAA.

${ }^{\ddagger}$ Associate Professor, Department of Aerospace Engineering, Associate Fellow AIAA

$\S$ PhD., Department of Aerospace Engineering 


$\begin{array}{ll}t & \text { time } \\ \boldsymbol{u} & \text { control vector } \\ u_{x} & \text { radial } \boldsymbol{i} \text {-component of control acceleration } \\ u_{y} & \text { tangential } \boldsymbol{j} \text {-component of control acceleration } \\ u_{z} & \text { normal } \boldsymbol{k} \text {-component of control acceleration } \\ \boldsymbol{x} & \text { state vector } \\ x & \text { radial } \boldsymbol{i} \text {-component of } \delta \boldsymbol{r} \\ y & \text { tangential } \boldsymbol{j} \text {-component of } \delta \boldsymbol{r} \\ z & \text { normal } \boldsymbol{k} \text {-component of } \delta \boldsymbol{r} \\ \Phi\left(t, t_{0}\right) & \text { state transition matrix } \\ \delta \boldsymbol{r} & {[x y z]^{T}, \text { position vector of the spacecraft from the origin of the rotating frame }} \\ \mu & \text { gravitational parameter of the central body } \\ \boldsymbol{\omega} & \omega \boldsymbol{k}=\sqrt{\mu / R^{3}} \boldsymbol{k}, \text { constant angular velocity vector } \\ |\cdot| & \text { magnitude of the vector quantity } \cdot \\ & \\ \text { Subscripts } & \\ f & \text { terminal value of the variable } \\ o & \text { initial value of the variable }\end{array}$

\author{
Superscripts \\ - $\quad$ time derivative \\ * $\quad$ optimized or minimized variable $(\cdot)$ with respect to $\boldsymbol{u}$
}

\title{
I. Introduction
}

This paper presents a novel approach to evaluating optimal continuous thrust trajectories and feedback control laws for a spacecraft subject to a general gravity field. This approach is derived by relying on the Hamiltonian nature of the necessary conditions associated with optimal control, and by utilizing certain properties of generating functions and canonical transformations. In particular, we show that certain solutions to the Hamilton-Jacobi (HJ) equation, associated with canonical transformations of Hamiltonian systems, can directly yield optimal control laws for a general system. Typically, application of Pontryagin's principle changes the nonlinear optimal rendezvous problem to a two point boundary value problem (TPBVP), for which one generally requires an initial estimate for the initial (or final) adjoint variables followed by an iterative solution procedure. Our approach provides an algorithm to compute the initial (or final) values of the adjoints without requiring an initial estimate, and for arbitrary boundary conditions, simply by algebraic manipulations of the generating function. Our approach not only satisfies the TPBVP found from the necessary conditions, by definition, but also satisfies the Hamilton-Jacobi-Bellman equation for the optimal cost, which is a sufficient condition for optimality. Most importantly, we have derived and applied a general solution procedure for this problem to a non-linear dynamical system of interest to astrodynamics. To develop and apply this algorithm requires certain conditions on the dynamics and cost function, which we detail in this paper.

Since Lawden ${ }^{1}$ initially introduced primer vector theory, the problem of continuous thrust optimal rendezvous has been a topic of continual interest. Much work has been done on this topic, so in the following we only give a brief review of work that has direct relation to analytical work on the optimal rendezvous problem for space trajectories. Billik $^{2}$ applied differential game theory to rendezvous problems subject to linearized dynamics. London ${ }^{3}$ and Antony and Sasaki ${ }^{4}$ studied the uncontrolled motion subject to second order approximation. Euler ${ }^{5}$ considered low-thrust optimal rendezvous maneuver in the vicinity of an elliptical orbit. Jezewski and Stoolz ${ }^{6}$ considered minimum-time problems subject to the inverse square field and evaluated an analytic solution under highly restricted assumptions. Later, Marec ${ }^{7}$ extended Lawden's primer vector theory graphically with the Contensou principle. Various types of continuous thrust optimal rendezvous problems subject to a linearized gravity field have been extensively explored by

2 of 22 
Carter, Humi, Paridis, etc ${ }^{8,9,10,11}$. Also, Lembeck and Prussing ${ }^{12}$ solved a combined problem of impulse intercept and continuous-thrust rendezvous subject to linearized dynamics.

As is seen, except for the very basic works of London, Antony et al, and Jezewski et al, all of the above researches consider linearized dynamics, which clearly limits the applicability and utility of this problem. Thus it is desirable to find the optimal trajectory subject to the original nonlinear dynamics. However, to do so in general requires one to solve the TPBVP for the adjoints for each boundary condition of interest, a challenging problem. Additionally, it is even more difficult to find a non-linear optimal feedback control, generally found by solving the Hamilton-JacobiBellman (HJB) equation for the optimal cost function.

Recently using generating functions appearing in Hamilton-Jacobi theory, Guibout and Scheeres ${ }^{13,14}$ suggested a new methodology to solve TPBVPs for Hamiltonian systems, including optimal control systems defined by Pontryagin's necessary condition. Based on these works, Park and Scheeres ${ }^{15}$ devised a new algorithm to solve optimal feedback control problem. Evaluating generating functions, they computed the initial adjoints (without guess) and obtained optimal feedback control for a special type of boundary conditions (hard constraint problem). Then Scheeres et al. ${ }^{16}$ applied their method to the non-linear optimal rendezvous of a spacecraft, and demonstrated that their higher order control law is superior to the linear control law. Later in [17] and [18] they extended the applicability of their method for general boundary conditions.

This document is a continuation and extension of [16] and demonstrates a direct application of this algorithm to continuous thrust optimal rendezvous problems subject to inverse-square central gravity fields. The discussion is structured as follows. First we give a brief review of classical optimal control theory as applied to a specific class of problems, then motivate our current approach and show how it satisfies the necessary conditions by default, and how it can be used to derive an optimal feedback control law. Then we formulate the continuous thrust optimal control of transferring from one state to another, using non-linear dynamics relative to a circular orbit. A detailed solution procedure follows, and the trajectories based on higher-order control law are compared with those based on linear control. Finally, we discuss the uniqueness of our solutions and contrast the current results with previous works.

\section{A General Solution of the Optimal Control Problem}

\section{A. Classical Necessary Conditions for Optimal Control}

Assume we have a dynamical system stipulated as $\dot{\boldsymbol{x}}=\boldsymbol{f}(\boldsymbol{x}, \boldsymbol{u}, t)$. The goal is to transfer from an initial state to a final state in a specified time span while minimizing some cost function. The application envisioned is for a spacecraft in a specified state (consisting of a specific orbit, hence position and velocity) to transition to another state while minimizing the Lagrange type cost function of the form:

$$
J=\int_{t_{o}}^{t_{f}} L(\boldsymbol{x}, \boldsymbol{u}, t) d t
$$

For a comprehensive introduction to the theory of optimal control of space trajectories we cite [1] and [7]. The Hamiltonian of the system can be stated as

$$
\mathcal{H}(\boldsymbol{x}, \boldsymbol{p}, \boldsymbol{u}, t)=L(\boldsymbol{x}, \boldsymbol{u}, t)+\boldsymbol{p}(t) \cdot \boldsymbol{f}(\boldsymbol{x}, \boldsymbol{u}, t) .
$$

Applying the Pontryagin principle we find the optimal control:

$$
\boldsymbol{u}^{*}=\arg \min _{\boldsymbol{u}} \mathcal{H}(\boldsymbol{x}, \boldsymbol{p}, \boldsymbol{u}, t)
$$

Substituting this control back into $\mathcal{H}$ leads to the new Hamiltonian $\mathcal{H}^{*}(\boldsymbol{x}, \boldsymbol{p}, t)$ and to a necessary condition for the optimal control system:

$$
\begin{aligned}
\dot{\boldsymbol{x}} & =\frac{\partial \mathcal{H}^{*}}{\partial \boldsymbol{p}} \\
\dot{\boldsymbol{p}} & =-\frac{\partial \mathcal{H}^{*}}{\partial \boldsymbol{x}}
\end{aligned}
$$


For the associated boundary conditions, note that the initial and terminal states should be completely specified to reflect the rendezvous condition, i.e.,

$$
\boldsymbol{x}\left(t_{o}\right)=\boldsymbol{x}_{o}, \boldsymbol{x}\left(t_{f}\right)=\boldsymbol{x}_{f}
$$

The fundamental difficulty in this approach is, as is well known, finding the initial or final adjoints, $\boldsymbol{p}_{o}$ or $\boldsymbol{p}_{f}$, satisfying this boundary value problem. Once we find $\boldsymbol{p}_{o}$ ( or $\boldsymbol{p}_{f}$ ), we can directly integrate the associated differential equations forward (or backward), along with the specified initial (or terminal) states, solving for the optimal control from the Pontryagin's principle at each point along the trajectory.

\section{B. Motivation of the Proposed Method}

The drawback of the approach described above is that solution procedures for the TPBVP generally require an initial estimate for the adjoints, which usually have no physical interpretations. Furthermore, we must repetitively solve the TPBVP for each boundary condition of interest, which is time-consuming, lacks definiteness, and is subject to numerical divergence. The conventional alternative method is to solve the Hamilton-Jacobi-Bellman (HJB) equation for the optimal cost function, and thus to evaluate the optimal cost and the corresponding optimal control law. However, the HJB is a first order partial differential equation and is extremely difficult to solve in general. Furthermore, for the boundary condition we are considering here, the HJB cost function has a singularity at the terminal condition, which makes the problem even more difficult (This singularity is discussed in more detail in [15]).

In an attempt to overcome these disadvantages, we suggest an alternative method, which specifically utilizes the theory of canonical transformations and their associated generating functions. This method provides a way to compute the initial (or final) adjoints as a function of known initial and/or final states, and thus to evaluate the optimal trajectory by simple forward (or backward) integration. It also enables us to systematically construct the optimal feedback control, even with the fundamental singularity prevailing in the HJB equation at its terminal condition. The next section is dedicated to the discussion of our approach.

\section{Solution of the Boundary Value Problem using Generating Functions}

Recall the theory of canonical transformations and generating functions in Hamiltonian dynamics (c.f. [19]). In addition to generating canonical transformations between Hamiltonian systems, generating functions also solve boundary value problems between Hamiltonian coordinate and momentum states for a single flow field. See the Appendix for a more detailed derivation of the results we present in the following.

In the case where the initial and terminal states are explicitly given, the generating function $F_{1}\left(\boldsymbol{x}_{o}, \boldsymbol{x}_{f}, t_{o}, t_{f}\right)$ can be directly used to find the initial and final momentum vectors from the relationship:

$$
\boldsymbol{p}_{o}=-\frac{\partial F_{1}\left(\boldsymbol{x}_{o}, \boldsymbol{x}_{f}, t_{o}, t_{f}\right)}{\partial \boldsymbol{x}_{o}} ; \boldsymbol{p}_{f}=\frac{\partial F_{1}\left(\boldsymbol{x}_{o}, \boldsymbol{x}_{f}, t_{o}, t_{f}\right)}{\partial \boldsymbol{x}_{f}}
$$

The generating function $F_{1}$ also satisfies a Hamilton-Jacobi partial differential equation:

$$
\frac{\partial F_{1}}{\partial t_{f}}+\mathcal{H}^{*}\left(\boldsymbol{x}_{f}, \frac{\partial F_{1}}{\partial \boldsymbol{x}_{f}}, t_{f}\right)=0
$$

Analogous relations and definitions exist for the generating functions $F_{2}\left(\boldsymbol{x}_{f}, \boldsymbol{p}_{o}, t_{o}, t_{f}\right), F_{3}\left(\boldsymbol{x}_{o}, \boldsymbol{p}_{f}, t_{o}, t_{f}\right)$, and $F_{4}\left(\boldsymbol{p}_{o}, \boldsymbol{p}_{f}, t_{o}, t_{f}\right)$ with results:

$$
\begin{gathered}
\boldsymbol{x}_{o}=\frac{\partial F_{2}}{\partial \boldsymbol{p}_{o}} \quad ; \quad \boldsymbol{p}_{f}=\frac{\partial F_{2}}{\partial \boldsymbol{x}_{f}} \\
\boldsymbol{p}_{o}=-\frac{\partial F_{3}}{\partial \boldsymbol{x}_{o}} \quad ; \quad \boldsymbol{x}_{f}=-\frac{\partial F_{3}}{\partial \boldsymbol{p}_{f}} \\
\boldsymbol{x}_{o}=-\frac{\partial F_{4}}{\partial \boldsymbol{p}_{o}} \quad ; \quad \boldsymbol{x}_{f}=\frac{\partial F_{4}}{\partial \boldsymbol{p}_{f}}
\end{gathered}
$$


These functions all solve their own version of the Hamilton-Jacobi equation:

$$
\begin{aligned}
\frac{\partial F_{2}}{\partial t_{f}}+\mathcal{H}^{*}\left(\boldsymbol{x}_{f}, \frac{\partial F_{2}}{\partial \boldsymbol{x}_{f}}, t_{f}\right) & =0 \\
\frac{\partial F_{3}}{\partial t_{f}}+\mathcal{H}^{*}\left(-\frac{\partial F_{3}}{\partial \boldsymbol{p}_{f}}, \boldsymbol{p}_{f}, t_{f}\right) & =0 \\
\frac{\partial F_{4}}{\partial t_{f}}+\mathcal{H}^{*}\left(-\frac{\partial F_{4}}{\partial \boldsymbol{p}_{f}}, \boldsymbol{p}_{f}, t_{f}\right) & =0
\end{aligned}
$$

A final property of the generating functions is that they can be transformed into each other via the Legendre transformation. Specifically, we find the following relations between the generating functions:

$$
\begin{aligned}
& F_{2}\left(\boldsymbol{x}_{o}, \boldsymbol{p}_{f}, t_{o}, t_{f}\right)=F_{1}\left(\boldsymbol{x}_{o}, \boldsymbol{x}_{f}, t_{o}, t_{f}\right)+\boldsymbol{p}_{f} \cdot \boldsymbol{x}_{f} \\
& F_{3}\left(\boldsymbol{p}_{o}, \boldsymbol{x}_{f}, t_{o}, t_{f}\right)=F_{1}\left(\boldsymbol{x}_{o}, \boldsymbol{x}_{f}, t_{o}, t_{f}\right)-\boldsymbol{p}_{o} \cdot \boldsymbol{x}_{o} \\
& F_{4}\left(\boldsymbol{p}_{o}, \boldsymbol{p}_{f}, t_{o}, t_{f}\right)=F_{2}\left(\boldsymbol{x}_{o}, \boldsymbol{p}_{f}, t_{o}, t_{f}\right)-\boldsymbol{p}_{o} \cdot \boldsymbol{x}_{o}
\end{aligned}
$$

The key observation we make is that solving for $F_{1}$ solves the boundary value problem and hence the optimal control problem. Suppose there exists an analytical form for $F_{1}$ such that we can find it. Then, we can directly take its partial derivatives, specify $\boldsymbol{x}_{o}$ and $\boldsymbol{x}_{f}$, and find the appropriate momentum to generate the optimal control for rendezvous. Also it has been shown that $J=-F_{1}$ satisfies the Hamilton-Jacobi-Bellman equation and thus is the optimal cost function and satisfies the sufficient condition for optimality ${ }^{18}$. Finally using (3) and the desired $F_{1}$ function we can define a feedback control law:

$$
\boldsymbol{u}^{*}=\arg \min _{u} \mathcal{H}\left[\boldsymbol{x},-\frac{\partial F_{1}\left(\boldsymbol{x}, \boldsymbol{x}_{f}, t, t_{f}\right)}{\partial \boldsymbol{x}}, \boldsymbol{u}, t\right]
$$

where we fix the terminal boundary condition $\boldsymbol{x}_{f}$ and allow the initial condition to equal the current state. Note that we have replaced $\boldsymbol{x}_{o}$ and $t_{o}$ with $\boldsymbol{x}$ and $t$ to stress the arbitrariness of initial states, and thus the feedback nature of the control law.

\section{Implementing a solution for $F_{1}$}

The difficulty, of course, is in finding the generating function $F_{1}$. This problem is directly addressed in Guibout and Scheeres ${ }^{14}$, where they show that the generating functions, if they exist in analytical form, can be solved as power series expansions in their respective arguments. The coefficients of these power series satisfying a set of ordinary differential equations derived from the Hamilton-Jacobi equations for generating functions.

To carry out this method, however, requires that some restrictions be placed on the system dynamics and cost function. The approach developed in [14] is based on constructing local solutions to the generating functions, i.e., expanding them as a Taylor series about a nominal trajectory that is known. This implies that a solution to the optimal control problem has already been found, and our specific method operates in the vicinity of this solution. It is important to note that this includes the case of no control, i.e., if the dynamics of the system carry a state between two points, $\boldsymbol{x}_{o}$ to $\boldsymbol{x}_{f}$, then the optimal control for this transition is simply stated as $\boldsymbol{u} \equiv 0$, and our method can be used on such a system.

Thus, to formally apply that method to the current system requires that the system has zero equilibrium point and satisfy $\boldsymbol{f}(\boldsymbol{x}=0, \boldsymbol{u}=0, t)=\mathbf{0}$. Furthermore, as we expand the Hamiltonian as a Taylor series of states and adjoints about a nominal solution, we also require analyticity of Hamiltonian. This, in turn, places a requirement on the analyticity of $L$ in (1) (since this becomes part of the Hamiltonian function through the Pontryagin principle). Finally, we assume the control $u$ is unbounded also for the sake of analyticity ${ }^{\mathrm{a}}$.

${ }^{a}$ It should be noted that even with all these strong assumptions about analyticity, the convergence of the series solution of generating function is not always guaranteed. For some special cases including resonance phenomenon, the convergence of the series solution may be suspect as time evolves, in which case our series-based method should be used with caution. 
The process derived in [14] consists of expanding the Hamiltonian function as a Taylor series in the states and adjoints, and the $F_{1}$ generating function as Taylor series in the initial and final states. Then the series for $F_{1}$ is substituted into the Hamilton-Jacobi equation (8) and a balancing technique is used to equate all like powers of the states to zero. This defines a set of differential equations for the coefficients of the $F_{1}$ Taylor series expansion. A major problem in this approach, however, is that initial conditions for $F_{1}$ at time $t_{o}=t_{f}$ and $\boldsymbol{x}_{o} \neq \boldsymbol{x}_{f}$ are undefined, making it impossible to initiate the integration of the coefficients. Furthermore, these coefficients are not known $a$ priori at any other time. This problem can be circumvented, however, by solving for a different generating function and then transforming back to the $F_{1}$ function, using the Legendre transformation, at some later moment when $F_{1}$ is well defined ${ }^{\mathrm{b}}$. Given a power series expansion for a given $F_{i}, i=1 \sim 4$, it is always possible to transform to a different generating function using the transformations in (15)-(17) along with the fundamental results given in (7), (9)-(11).

The $F_{2}$ generating function, it turns out, can be solved using our initial value approach, contrast to $F_{1}$. This is due to the fact that $F_{2}$ can generate the identity transformation when $t_{o}=t_{f}{ }^{\mathrm{c}}$. Thus, we solve for the $F_{2}$ generating function as a function of time by integrating the differential equations for the coefficients and, when needed, transform to the $F_{1}$ function via the Legendre transformation to solve the boundary value problem, which in turn solves the optimal control problem.

In this paper we investigate the application of this approach to the optimal control of a spacecraft in the vicinity of a nominal trajectory, incorporating dynamical non-linearities. For definiteness we will develop and apply this approach for a specific example.

\section{A Specific Formulation of the Optimal Rendezvous Problem}

Consider a spacecraft subject to a central gravity field. Its equations of motion, in the inertial frame with the origin located at the center of gravity, are given by

$$
\ddot{\boldsymbol{r}}=-\frac{\mu}{r^{3}} \boldsymbol{r}+\frac{\boldsymbol{F}}{m}
$$

We introduce another coordinate frame which is rotating along a circular orbit at a constant angular velocity. Then represented in the rotating coordinate frame, the position, velocity, and acceleration vectors are, respectively,

$$
\begin{aligned}
\boldsymbol{r} & =\boldsymbol{R}+\delta \boldsymbol{r}=(R+x) \boldsymbol{i}+y \boldsymbol{j}+z \boldsymbol{k} \\
\Rightarrow \dot{\boldsymbol{r}} & =(\dot{x}-\omega y) \boldsymbol{i}+[\dot{y}+\omega(R+x)] \boldsymbol{j}+\dot{z} \boldsymbol{k} \\
\Rightarrow \ddot{\boldsymbol{r}} & =\left[\ddot{x}-2 \omega \dot{y}-\omega^{2}(R+x)\right] \boldsymbol{i}+\left(\ddot{y}+2 \omega \dot{x}-\omega^{2} y\right) \boldsymbol{j}+\ddot{z} \boldsymbol{k} .
\end{aligned}
$$

From Newton's law, we obtain the following component-wise equations of motion in the rotating frame:

$$
\begin{aligned}
\ddot{x}-2 \omega \dot{y}-\omega^{2}(R+x) & =-\frac{\mu}{r^{3}}(R+x)+u_{x} \\
\ddot{y}+2 \omega \dot{x}-\omega^{2} y & =-\frac{\mu}{r^{3}} y+u_{y} \\
\ddot{z} & =-\frac{\mu}{r^{3}} z+u_{z}
\end{aligned}
$$

where $r=\sqrt{(R+x)^{2}+y^{2}+z^{2}}$. If non-dimensionalized with reference length $R$ and reference time $1 / \omega$, they are simplified as

$$
\ddot{x}-2 \dot{y}+(1+x)\left(\frac{1}{r^{3}}-1\right)=u_{x}
$$

\footnotetext{
${ }^{b}$ In general, it happens that all generating functions may suffer from singularities, but at different moments. In this case it is impossible to solve for one generating function for the entire time span of interest. Instead, we initiate the time evolution for one generating function, then before a singularity occurs, we jump to another generating function via the Legendre transformation to re-initiate the time evolution. However, it will be shown later that $F_{1}$ and $F_{2}$ in our formulation do not suffer from such multiple singularities, but have only one inherent singularity for $F_{1}$. For those who are more interested in this singularity issue, we cite Guibout and Scheeres ${ }^{13}$.

${ }^{\mathrm{c}}$ Refer to [13] and [18] for more detailed arguments.
} 


$$
\begin{aligned}
\ddot{y}+2 \dot{x}+y\left(\frac{1}{r^{3}}-1\right) & =u_{y} \\
\ddot{z}+\frac{1}{r^{3}} z & =u_{z}
\end{aligned}
$$

where now $r=\sqrt{(x+1)^{2}+y^{2}+z^{2}}$. For simplicity's sake, we consider planar motion henceforth. Defining the states as $\boldsymbol{x}=\left[\begin{array}{llll}x_{1} & x_{2} & x_{3} & x_{4}\end{array}\right]^{T}=\left[\begin{array}{llll}x & y & \dot{x} & \dot{y}\end{array}\right]^{T}$ and control as $\boldsymbol{u}=\left[\begin{array}{ll}u_{1} & u_{2}\end{array}\right]^{T}=\left[\begin{array}{ll}u_{x} & u_{y}\end{array}\right]^{T}$, we can construct the equations of planar motion in state space form:

$$
\left[\begin{array}{c}
\dot{x}_{1} \\
\dot{x}_{2} \\
\dot{x}_{3} \\
\dot{x}_{4}
\end{array}\right]=\left[\begin{array}{c}
x_{3} \\
x_{4} \\
2 x_{4}-\left(1+x_{1}\right)\left(\frac{1}{r^{3}}-1\right)+u_{1} \\
-2 x_{3}-x_{2}\left(\frac{1}{r^{3}}-1\right)+u_{2}
\end{array}\right]
$$

where $r=\sqrt{\left(x_{1}+1\right)^{2}+x_{2}^{2}}$. Note that linearization about the circular reference trajectory leads to the in-plane dynamics of the well-known Clohessy-Wiltshire equation:

$$
\begin{aligned}
& {\left[\begin{array}{c}
\dot{x}_{1} \\
\dot{x}_{2} \\
\dot{x}_{3} \\
\dot{x}_{4}
\end{array}\right]=\left[\begin{array}{cccc}
0 & 0 & 1 & 0 \\
0 & 0 & 0 & 1 \\
3 & 0 & 0 & 2 \\
0 & 0 & -2 & 0
\end{array}\right]\left[\begin{array}{l}
x_{1} \\
x_{2} \\
x_{3} \\
x_{4}
\end{array}\right]+\left[\begin{array}{ll}
0 & 0 \\
0 & 0 \\
1 & 0 \\
0 & 1
\end{array}\right]\left[\begin{array}{l}
u_{1} \\
u_{2}
\end{array}\right]} \\
& \Leftrightarrow \dot{x}=A x+B u
\end{aligned}
$$

Furthermore the right hand side of (29) is analytic and can be expanded as a Taylor series about the circular reference trajectory:

$$
\left[\begin{array}{c}
\dot{x}_{1} \\
\dot{x}_{2} \\
\dot{x}_{3} \\
\dot{x}_{4}
\end{array}\right]=\left[\begin{array}{c}
x_{3} \\
x_{4} \\
3 x_{1}+2 x_{4}-3 x_{1}^{2}+1.5 x_{2}^{2}+4 x_{1}^{3}-6 x_{1} x_{2}^{2}+\cdots+u_{1} \\
-2 x_{3}+3 x_{1} x_{2}-6 x_{1}^{2} x_{2}+1.5 x_{2}^{3}+\cdots+u_{2}
\end{array}\right],
$$

a result which will be used later.

Finally, the objective is to minimize the quadratic performance index

$$
J=\frac{1}{2} \int_{t_{0}}^{t_{f}} \boldsymbol{u}^{T}(t) \boldsymbol{u}(t) d t
$$

subject to the nonlinear dynamics (29), satisfying given boundary conditions. Note that the integrand of $J$, i.e., $L=\boldsymbol{u}^{T} \boldsymbol{u} / 2=\left(u_{1}^{2}+u_{2}^{2}\right) / 2$, is analytic with respect to its arguments.

\section{A Non-linear Analytical Solution to the Optimal Rendezvous Problem}

\section{A. Derivation of the Optimal Solution in Series Form}

To summarize the previous discussion, let us consider minimization of the quadratic cost

$$
J=\frac{1}{2} \int_{t_{0}}^{t_{f}} \boldsymbol{u}^{T}(t) \boldsymbol{u}(t) d t
$$


subject to the system dynamics in central gravity field

$$
\left[\begin{array}{c}
\dot{x}_{1} \\
\dot{x}_{2} \\
\dot{x}_{3} \\
\dot{x}_{4}
\end{array}\right]=\left[\begin{array}{c}
x_{3} \\
x_{4} \\
3 x_{1}+2 x_{4}-3 x_{1}^{2}+1.5 x_{2}^{2}+4 x_{1}^{3}-6 x_{1} x_{2}^{2}+\cdots+u_{1} \\
-2 x_{3}+3 x_{1} x_{2}-6 x_{1}^{2} x_{2}+1.5 x_{2}^{3}+\cdots+u_{2}
\end{array}\right] .
$$

With the Hamiltonian $\mathcal{H}$ defined as

$$
\mathcal{H}=\frac{1}{2} \boldsymbol{u}^{T} \boldsymbol{u}+\boldsymbol{p}^{T} \dot{\boldsymbol{x}},
$$

which can be expanded as a Taylor series to find

$$
\begin{aligned}
\mathcal{H} & =\frac{1}{2}\left(u_{1}^{2}+u_{2}^{2}\right)+p_{1} x_{3}+p_{2} x_{4}+p_{3}\left(3 x_{1}+2 x_{4}-3 x_{1}^{2}+1.5 x_{2}^{2}+4 x_{1}^{3}-6 x_{1} x_{2}^{2}+\cdots+u_{1}\right) \\
& +p_{4}\left(-2 x_{3}+3 x_{1} x_{2}-6 x_{1}^{2} x_{2}+1.5 x_{2}^{3}+\cdots+u_{2}\right)
\end{aligned}
$$

the corresponding costate equations are

$$
\left[\begin{array}{c}
\dot{p}_{1} \\
\dot{p}_{2} \\
\dot{p}_{3} \\
\dot{p}_{4}
\end{array}\right]=\left[\begin{array}{c}
-3 p_{3}+6 x_{1} p_{3}-3 x_{2} p_{4}-12 x_{1}^{2} p_{3}+6 x_{2}^{2} p_{3}+12 x_{1} x_{2} p_{4} \cdots \\
-3 x_{2} p_{3}-3 x_{1} p_{4}+12 x_{1} x_{2} p_{3}+6 x_{1}^{2} p_{4}-4.5 x_{2}^{2} p_{4} \cdots \\
-p_{1}+2 p_{4} \\
-p_{2}-2 p_{3}
\end{array}\right]
$$

From the optimality condition $\mathcal{H}_{u}=0$ we find the optimal control law:

$$
\left[\begin{array}{l}
u_{1} \\
u_{2}
\end{array}\right]=\left[\begin{array}{l}
-p_{3} \\
-p_{4}
\end{array}\right]
$$

Introducing (38) into (36) yields the Hamiltonian as a function of states and adjoints:

$$
\begin{aligned}
\mathcal{H}(\boldsymbol{x}, \boldsymbol{p}, t)= & \frac{1}{2}\left(p_{3}^{2}+p_{4}^{2}\right)+p_{1} x_{3}+p_{2} x_{4}+p_{3}\left(3 x_{1}+2 x_{4}-3 x_{1}^{2}+1.5 x_{2}^{2}+4 x_{1}^{3}-6 x_{1} x_{2}^{2}+\cdots-p_{3}\right) \\
& +p_{4}\left(-2 x_{3}+3 x_{1} x_{2}-6 x_{1}^{2} x_{2}+1.5 x_{2}^{3}+\cdots-p_{4}\right)
\end{aligned}
$$

As discussed earlier, we evaluate $F_{2}\left(\boldsymbol{x}, \boldsymbol{p}_{o}, t ; t_{0}\right)$ as a power series instead of $F_{1}\left(\boldsymbol{x}, \boldsymbol{x}_{o}, t ; t_{0}\right)$. For illustration purposes, in the following we only derive the equations to the linear order for the control law, in the actual analysis we kept terms up to higher orders using symbolic manipulators. The Hamiltonian is reduced to

$$
\mathcal{H}(\boldsymbol{x}, \boldsymbol{p}, t)=\frac{1}{2}\left[\begin{array}{l}
\boldsymbol{x} \\
\boldsymbol{p}
\end{array}\right]^{T}\left[\begin{array}{cc}
0 & A^{T} \\
A & -B B^{T}
\end{array}\right]\left[\begin{array}{l}
\boldsymbol{x} \\
\boldsymbol{p}
\end{array}\right]+\text { H.О.T }
$$

In keeping with this quadratic form of the Hamiltonian, we also expand $F_{2}$ in a quadratic form:

$$
F_{2}\left(\boldsymbol{x}, \boldsymbol{p}_{o}, t ; t_{0}\right)=\frac{1}{2}\left[\begin{array}{c}
\boldsymbol{x} \\
\boldsymbol{p}_{o}
\end{array}\right]^{T}\left[\begin{array}{cc}
F_{x x}\left(t ; t_{0}\right) & F_{x p_{o}}\left(t ; t_{0}\right) \\
F_{p_{o} x}\left(t ; t_{0}\right) & F_{p_{o} p_{o}}\left(t ; t_{0}\right)
\end{array}\right]\left[\begin{array}{c}
\boldsymbol{x} \\
\boldsymbol{p}_{o}
\end{array}\right]+\text { H.O.T }
$$

Now recalling the relation

$$
\boldsymbol{p}=\frac{\partial F_{2}}{\partial \boldsymbol{x}}=\left[\begin{array}{ll}
F_{x x} & F_{x p_{o}}
\end{array}\right]\left[\begin{array}{c}
\boldsymbol{x} \\
\boldsymbol{p}_{o}
\end{array}\right]+\text { H.O.T }
$$

$$
8 \text { of } 22
$$


we can express the Hamiltonian as

$$
\mathcal{H}=\frac{1}{2}\left[\begin{array}{c}
\boldsymbol{x} \\
\boldsymbol{p}_{o}
\end{array}\right]^{T}\left[\begin{array}{ll}
I & F_{x x} \\
0 & F_{p_{o} x}
\end{array}\right]\left[\begin{array}{cc}
0 & A^{T} \\
A & -B B^{T}
\end{array}\right]\left[\begin{array}{cc}
I & 0 \\
F_{x x} & F_{x p_{o}}
\end{array}\right]\left[\begin{array}{c}
\boldsymbol{x} \\
\boldsymbol{p}_{o}
\end{array}\right]+\text { H.O.T }
$$

Introduction of (41) and (43) into the HJ equation (12) yields the following set of differential equations for $F_{x x}\left(t ; t_{0}\right)$, $F_{x p_{o}}\left(t ; t_{0}\right)=F_{p_{o} x}^{T}\left(t ; t_{0}\right)$, and $F_{p_{o} p_{o}}\left(t ; t_{0}\right)$ :

$$
\begin{aligned}
0 & =\dot{F}_{x x}+F_{x x} A+A^{T} F_{x x}-F_{x x} B B^{T} F_{x x} \\
0 & =\dot{F}_{x p_{o}}+A^{T} F_{x p_{o}}-F_{x x} B B^{T} F_{x p_{o}} \\
0 & =\dot{F}_{p_{o} p_{o}}-F_{p_{o} x} B B^{T} F_{x p_{o}}
\end{aligned}
$$

Also, the corresponding initial conditions are derived from the identity transformation, $F_{2}\left(\boldsymbol{x}, \boldsymbol{p}_{o}, t=t_{0} ; t_{0}\right)=\boldsymbol{x} \cdot \boldsymbol{p}_{o}$, as ${ }^{\mathrm{d}}$

$$
\begin{aligned}
F_{x x}\left(t_{0} ; t_{0}\right) & =0 \\
F_{x p_{o}}\left(t_{0} ; t_{0}\right) & =I=F_{p_{o} x}\left(t_{0} ; t_{0}\right) \\
F_{p_{o} p_{o}}\left(t_{0} ; t_{0}\right) & =0 .
\end{aligned}
$$

Note that $F_{x x} \equiv 0$ due to the zero initial conditions; it satisfies the corresponding differential equation and the given initial condition. Generalizing this method, we can solve recursively for the remaining higher order terms. We do not show the higher order terms here, due to space limitations. The symbolic and numerical computations and results reported here have been carried out using Matlab ${ }^{\mathbb{R}}$ and Mathematica ${ }^{\circledR}$.

Once this system of differential equations is solved up to as high an order as desired, we can construct $F_{2}$. Then, rearranging the second equation of (9) for $\boldsymbol{p}_{f}=\boldsymbol{p}_{f}\left(\boldsymbol{x}_{0}, \boldsymbol{x}_{f}\right)$ using series inversion and introducing into the Legendre transformation (15) leads to $F_{1}\left(\boldsymbol{x}_{o}, \boldsymbol{x}_{f}, t_{o}, t_{f}\right)$ :

$$
\begin{aligned}
F_{1}= & \frac{1}{2}\left[\begin{array}{c}
\boldsymbol{x}_{f} \\
\boldsymbol{x}_{o}
\end{array}\right]^{T}\left[\begin{array}{cc}
\left(F_{x x}-F_{x p_{o}} F_{p_{o} p_{o}}^{-1} F_{p_{o} x}\right)\left(t_{f}, t_{o}\right) & \left(F_{x p_{o}} F_{p_{o} p_{o}}^{-1}\right)\left(t_{f}, t_{o}\right) \\
\left(F_{p_{o} p_{o}}^{-1} F_{p_{o} x}\right)\left(t_{f}, t_{o}\right) & -\left(F_{p_{o} p_{o}}^{-1}\right)\left(t_{f}, t_{o}\right)
\end{array}\right]\left[\begin{array}{l}
\boldsymbol{x}_{f} \\
\boldsymbol{x}_{o}
\end{array}\right] \\
& +\frac{1}{3 !} \sum_{i}^{2 n} \sum_{j}^{2 n} \sum_{k}^{2 n} f_{i j k}\left(t_{f}, t_{0}\right) y_{i} y_{j} y_{k}+\cdots
\end{aligned}
$$

where $\boldsymbol{y}=\left[\begin{array}{llll}y_{1} & y_{2} & \cdots & y_{2 n}\end{array}\right]^{T}=\left[\begin{array}{lll}x_{f 1} & \cdots & x_{f n} \\ x_{01} & \cdots & x_{0 n}\end{array}\right]^{T}$ and the last term indicates the higher order term expressed with a tensor notation ${ }^{\mathrm{e}}$. Note that the optimal cost function, from the HJB theory, simply equals $J\left(\boldsymbol{x}, t ; \boldsymbol{x}_{f}, t_{f}\right)=$ $-F_{1}\left(\boldsymbol{x}, \boldsymbol{x}_{f}, t, t_{f}\right)$, where we fix the terminal condition and take the initial condition as a moving coordinate $\left(\boldsymbol{x}_{o} \rightarrow\right.$ $\boldsymbol{x}(t)$ ). Also $\boldsymbol{p}_{o}$ can be computed from (7), which enables us to evaluate the optimal trajectory by simple forward integration. Finally, after some algebraic manipulations, the optimal feedback control can be obtained from (18):

$$
\boldsymbol{u}^{*}=-B^{T}\left[F_{p_{o} p_{o}}^{-1}\left(t_{f}, t\right)\left[\boldsymbol{x}(t)-F_{p_{o} x}\left(t_{f}, t\right) \boldsymbol{x}\left(t_{f}\right)\right]+\frac{\partial}{\partial \boldsymbol{x}} \frac{1}{3 !} \sum_{i}^{2 n} \sum_{j}^{2 n} \sum_{k}^{2 n} f_{i j k}\left(t_{f}, t\right) y_{i} y_{j} y_{k}+\cdots\right]
$$

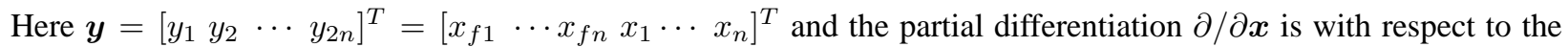
initial variables $\boldsymbol{x}=\left[\begin{array}{lll}x_{1} & \cdots & x_{n}\end{array}\right]^{T}=\left[\begin{array}{lll}y_{n+1} & \cdots & y_{2 n}\end{array}\right]^{T}$. Note that we only compute the coefficients for $F_{1}$ once as a function of time, then we have complete freedom to change initial and final conditions and time span.

Remark The above series solutions for $F_{1}$ and $F_{2}$ formally satisfy their respective HJ equations. Although our numerical comparisons with the reference trajectories are highly suggestive of the convergence of our series solution

\footnotetext{
${ }^{\mathrm{d}}$ Again, refer to [13] and [18] for more detailed arguments.

${ }^{\mathrm{e}}$ Refer to [13] for a rigorous derivation of higher order terms of $F_{1}$
} 
to the reference solution, we have not proven the convergence of our series solution and hence have not proven the existence of the solution. In Park and Scheeres ${ }^{17,18}$, it is proven that the feedback control law derived from $F_{1}$ satisfies the sufficient conditions for optimality. Thus, if the convergence of our series solution for $F_{1}$ is proven, then we will have satisfied the sufficient conditions. This is a topic of future research.

\section{B. Numerical Example}

Before discussing specific numerical examples, recall that we have used non-dimensionalized equations of motion. Given the appropriate scale factor, we can analyze any circular reference orbits of arbitrary altitude. Here, as an example, we consider a geosynchronous orbit where the reference frequency is $\omega=2 \pi / 1 \mathrm{day}=7.27 \times 10^{-5} \mathrm{rad} / \mathrm{sec}$, the reference time is $\bar{t}=1 / \omega=1.38 \times 10^{4} \mathrm{sec}$, and the reference length is $R=\sqrt[3]{\mu / \omega^{2}}=4.23 \times 10^{4} \mathrm{~km}$. Also for the control inputs, 1 non-dimensional unit corresponds to $0.222 \mathrm{~m} / \mathrm{sec}^{2}$.

Figures 1-9 show the optimal trajectory and control history for three specific examples. Example 1 (Figures 1-3) represents the result for a general offset in initial conditions of $[0.2,0.2,0.1,0.1]$ in position and velocity, transitioning to the origin $[0,0,0,0]$ in 1 unit of time. This rather general condition has been chosen to test the validity of our algorithm. Example 2 (Figures 4-6) shows the optimal trajectory starting from a circular orbit displaced in downtrack direction with an offset of 0.1 units, i.e., $[0,0.1,0,0]$, and then transitioning to a circular orbit at the coordinate frame origin in 1 unit of time. Example 3 (Figures 7-9) shows a similar result from an offset of 0.003 units, i.e., $[0,0.003,0,0]$, in the downtrack direction to the origin in $2 \pi$ units of time, i.e., one orbital period. As the time of the transfer increases, more terms are needed in the control to accurately approximate the true solution. Here the optimal controls are developed up to the 4th order.

If converted into real dimensions, Example 1 represent the transition from the initial condition of $[846 \mathrm{~km}, 846 \mathrm{~km}$, $0.307 \mathrm{~km} / \mathrm{sec}, 0.307 \mathrm{~km} / \mathrm{sec}$ ] to the origin in about 3.83hr. Similarly Examples 2 and 3, respectively, show the optimal transition from an initial offset of $4.23 \times 10^{3} \mathrm{~km}$ and $127 \mathrm{~km}$ in downtrack direction to a circular orbit at the coordinate frame origin in $3.83 \mathrm{hr}$ and 1 day, respectively.

For the control phase flows (Figures 3, 6, and 9), the solid line, dashed line, and dotted line indicate optimal trajectories computed from the original nonlinear systems, linearized systems about the reference orbit, and the 4th order approximated systems expanded as Taylor series about the reference orbit, respectively. The reference nonlinear solution (solid line) has been evaluated for comparison by solving the TPBVP numerically using a forward shooting method. The linear optimal control has been evaluated from the quadratic expansion of $F_{1}$ generating function, which also coincides with the solution from the Ricatti transformation method (or sweep method) in [20].

For the state trajectories (Figures 1, 2, 4, 5, 7, and 8), the solid line, dashed line, and dotted line represents the application of nonlinear, linear, and the 4th order control scheme to the original nonlinear system. It is clear that the 4th order control yields better approximation than the linear control. This observation also holds as long as the boundary condition is close enough to the reference trajectory.

Here note that $F_{1}$ and the associated feedback control law is only computed once and that each optimal trajectory is determined algebraically by introducing the appropriate numeric boundary condition, whereas the reference solution must be determined by solving TPBVP repetitively for the varying boundary conditions ${ }^{\mathrm{f}}$. By introducing additional higher order terms in the system dynamics, we can approximate the original system to as high an order as desired. The current implementation is limited only by computer memory constraints.

Figures 10-11 show the offset of the terminal boundary condition from the origin (that is, the true boundary condition to be satisfied) by the linear (dotted line) and 4th order control scheme (solid line). Here the initial conditions are chosen such that the initial positions are located along the circle of radius 0.15 and initial velocities are identically zero (that is, the initial conditions are $[0.15 \cos \theta 0.15 \sin \theta 00]$, with $\theta$ varying from 0 to $2 \pi$ radians). It is clear that for all phase angles the 4th order control scheme shows better convergent properties than the linear control scheme. Figures 12-13 show the phase trajectory of position variables and velocity variables for the same initial conditions. Finally Figure 14 shows the magnitude of control history. Again note that the initial conditions for all these results are

\footnotetext{
${ }^{\mathrm{f}}$ In fact, this favorable property suggests the following: given the same system we can obtain the optimal feedback control law for different types of boundary conditions without re-solving Hamilton-Jacobi partial differential equations. From the given $F_{1}$, we can compute feedback control law for other types of boundary conditions only by partial differentiation and series inversion. Refer to [17] for more details.
}

10 of 22 


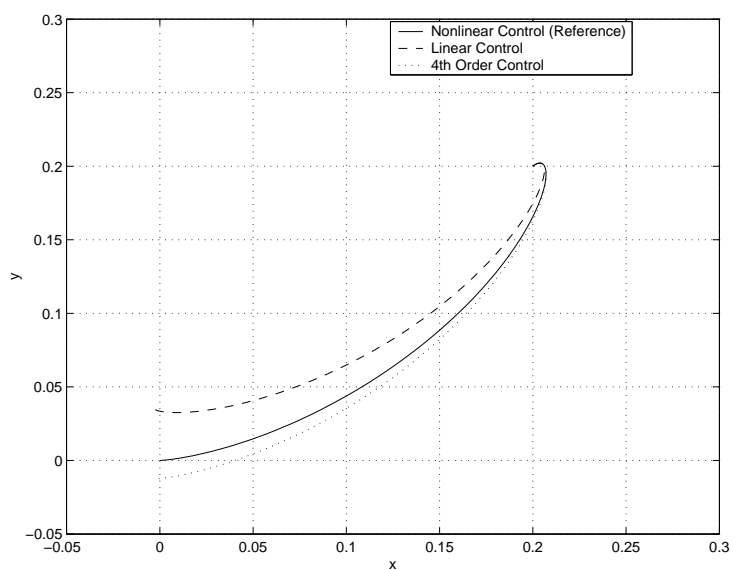

Figure 1. Radial and Tangential Positions (Example 1)

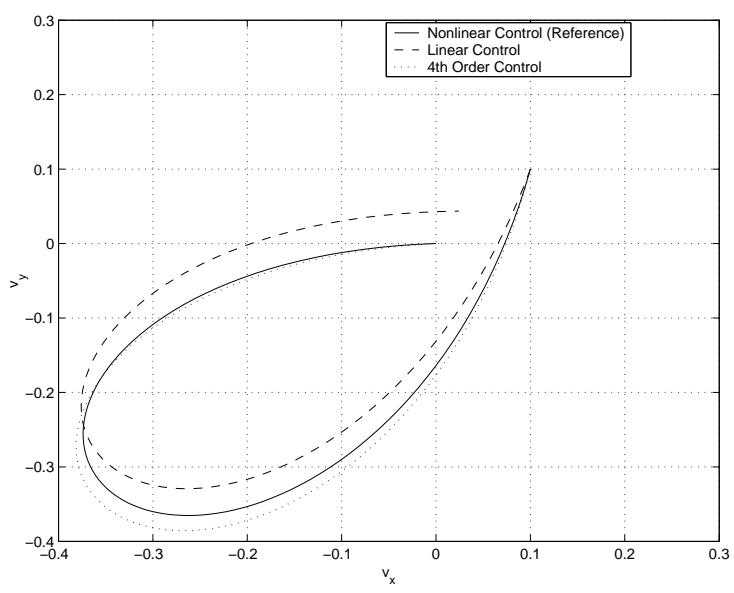

Figure 2. Radial and Tangential Velocities (Example 1)

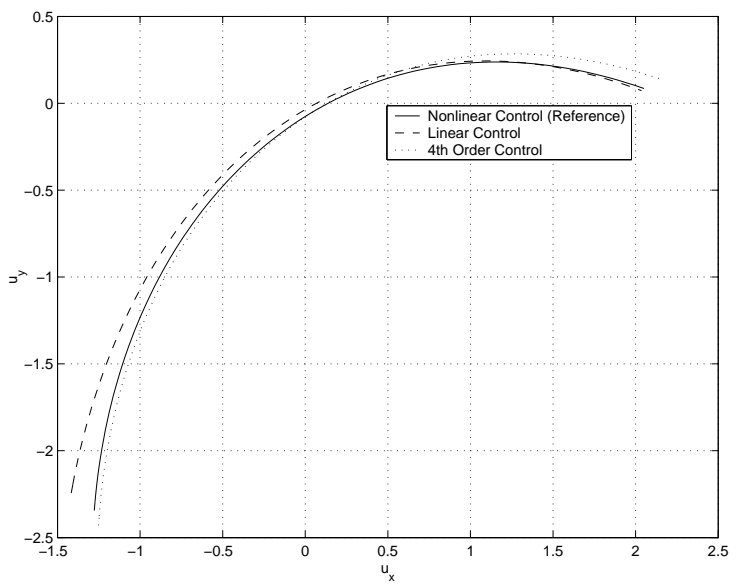

Figure 3. Radial and Tangential Controls (Example 1)

11 of 22

American Institute of Aeronautics and Astronautics 


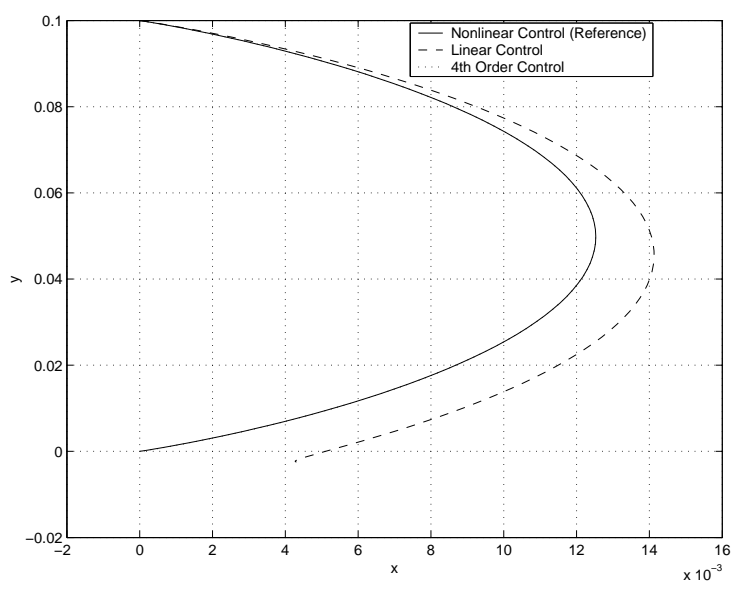

Figure 4. Radial and Tangential Positions (Example 2)

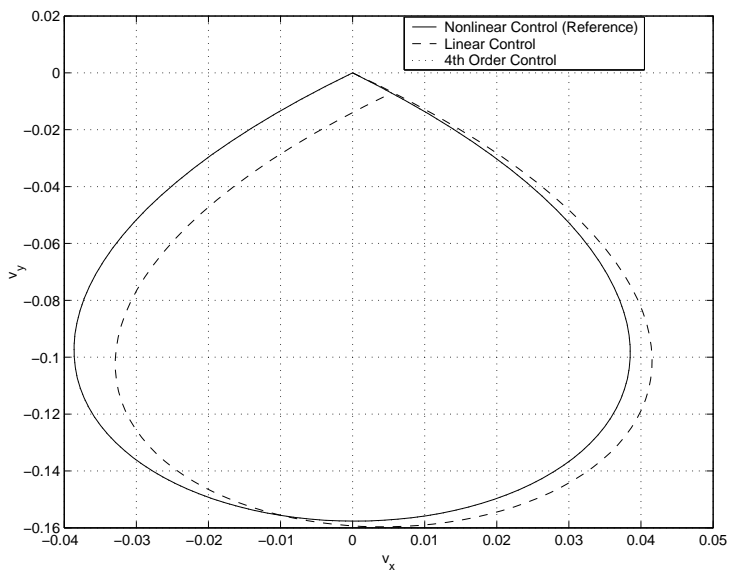

Figure 5. Radial and Tangential Velocities (Example 2)

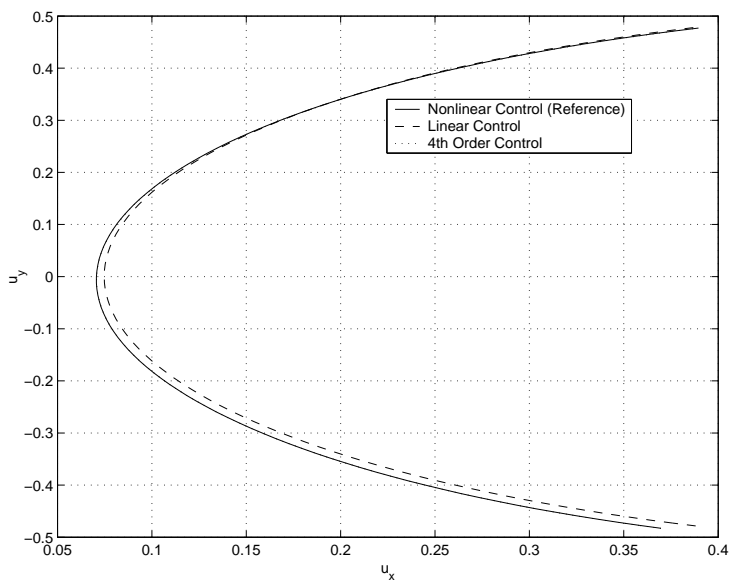

Figure 6. Radial and Tangential Controls (Example 2)

12 of 22

American Institute of Aeronautics and Astronautics 


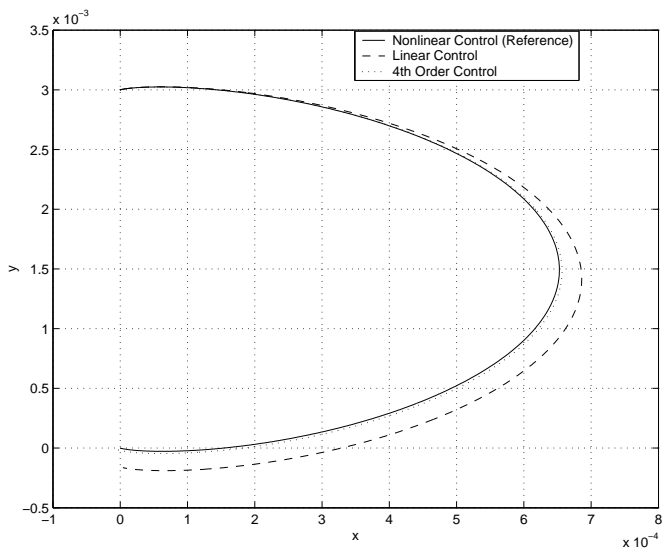

Figure 7. Radial and Tangential Positions (Example 3)

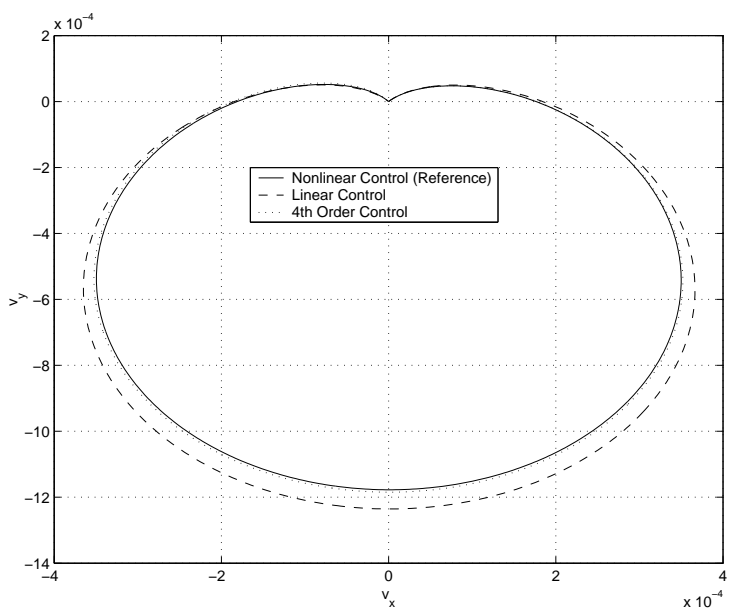

Figure 8. Radial and Tangential Velocities (Example 3)

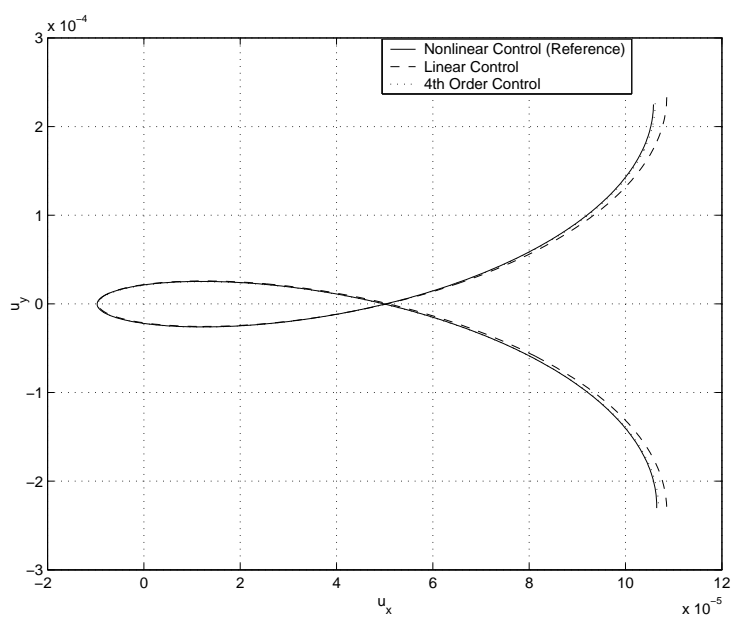

Figure 9. Radial and Tangential Controls (Example 3)

13 of 22

American Institute of Aeronautics and Astronautics 
obtained from the $F_{1}$ generating function which we only computed once; we have not solved the TPBVP numerically and repetitively.

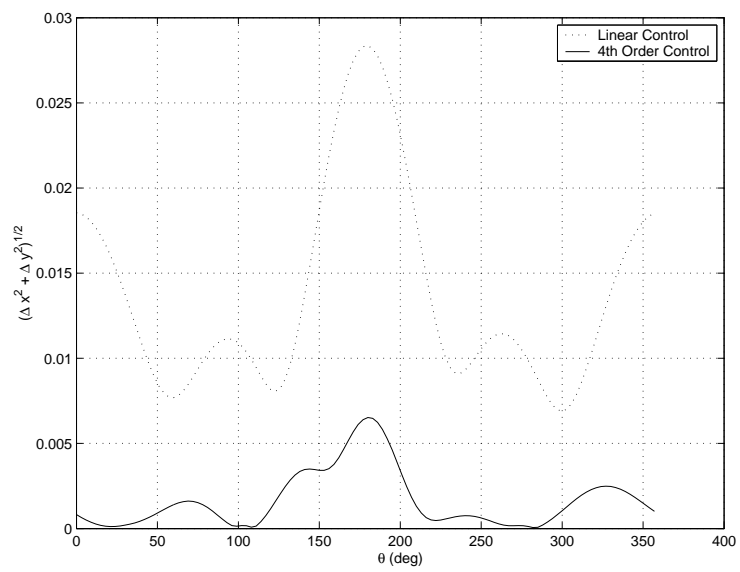

Figure 10. Terminal Position Offset $\boldsymbol{x}_{o}=[r \cos \theta r \sin \theta 00], r=0.15,0 \leq \theta \leq 360$ deg

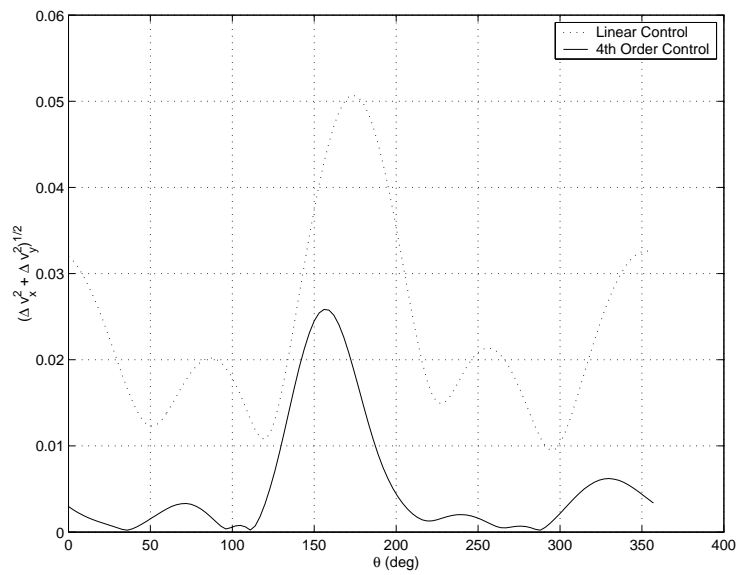

Figure 11. Terminal Velocity Offset $\boldsymbol{x}_{o}=[r \cos \theta r \sin \theta 00], r=0.15,0 \leq \theta \leq 360$ deg

\section{Singularities of Generating Functions}

So far we have demonstrated a step-by-step procedure for evaluating optimal trajectory as well as optimal feedback control via generating functions. Also it has been shown that once one kind of generating function is computed, the others can also be obtained by applying a Legendre transformation. This section is dedicated to a discussion on the possibility of singularities in the generating functions (and how to avoid them, if any) and their relationship to optimal trajectories. This is a potentially important issue. In [14] it was found that all the generating functions considered became "singular" at different times, and that this formed a fundamental barrier to the construction of long-term solutions for the generating functions (which was ultimately overcome). Thus, it is of interest to consider the possibility of singularities in the generating functions we are computing here.

In terms of the boundary value problem, presence of singularities are usually associated with the existence of multiple solutions to the problem. In the case of Lambert-type problems in astrodynamics, a familiar situation where this arises concerns $180^{\circ}$ transfers about a point-mass in a fixed time, as an infinity of possible transfer trajectories 


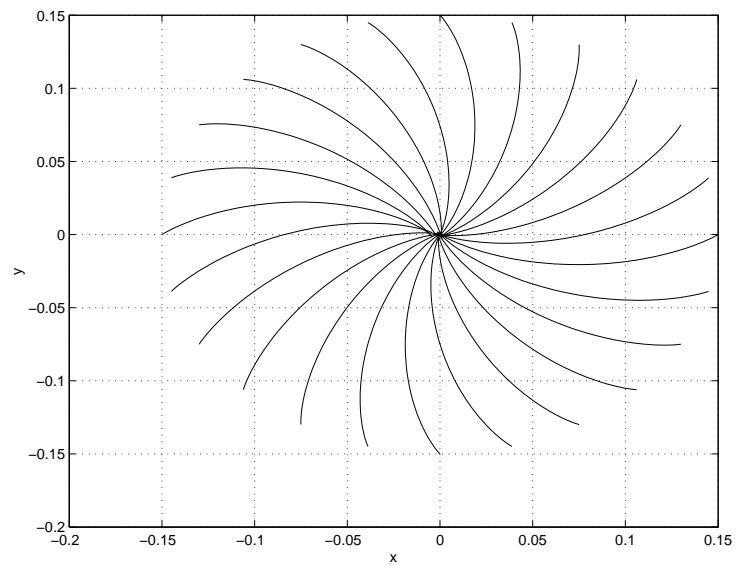

Figure 12. Positional Trajectory $\boldsymbol{x}_{o}=[r \cos \theta r \sin \theta 00], r=0.15,0 \leq \theta \leq 360$ deg

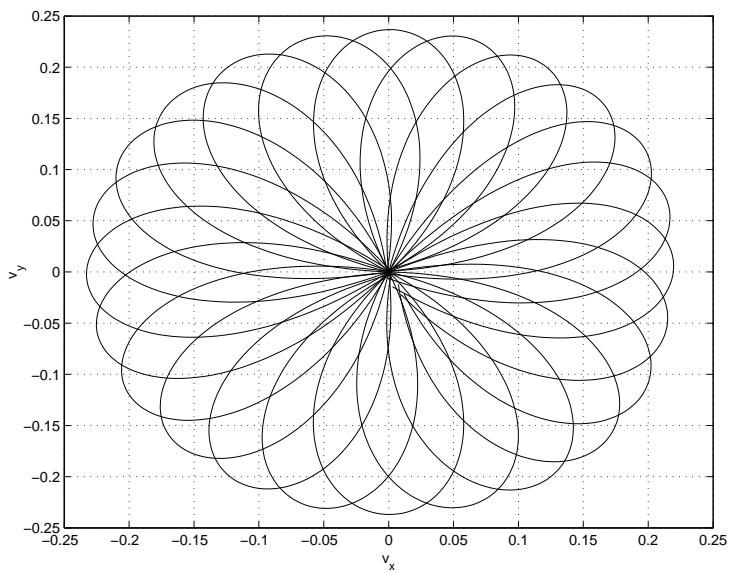

Figure 13. Velocity Trajectory $\boldsymbol{x}_{o}=[r \cos \theta r \sin \theta 00], r=0.15,0 \leq \theta \leq 360 d$ deg

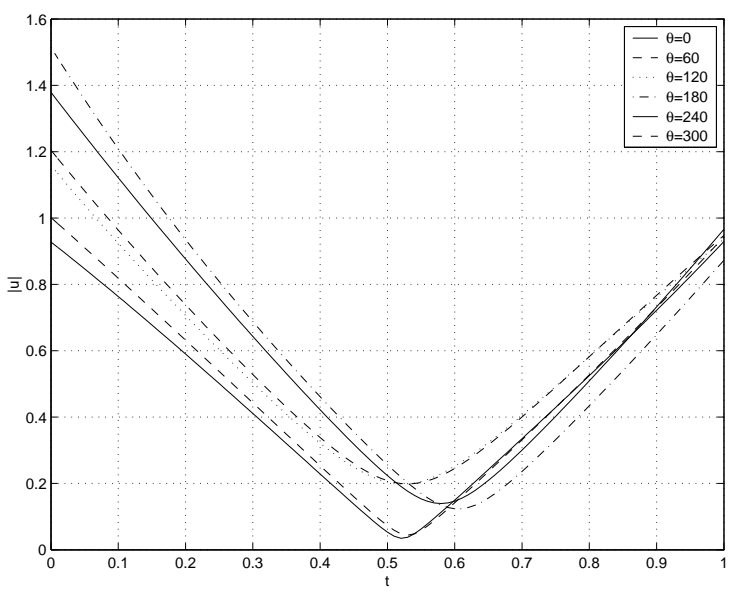

Figure 14. Control History $\boldsymbol{x}_{o}=[r \cos \theta r \sin \theta 00], r=0.15,0 \leq \theta \leq 360 \mathrm{deg}$

15 of 22

American Institute of Aeronautics and Astronautics 
exist. The singularities arise in our approach as soon as there is more than one possible solution to the boundary value problem, as then the linear order terms in our expansion for $F_{i}$ become degenerate and cannot represent the true solutions. This leads to a divergence in these linear terms, and serves as a barrier for continued integration of the coefficients. It is important to note, however, that not all the generating functions can become singular at one time, and thus it is always possible to transform to a different generating function using the Legendre transformation, and continue computation of that generating function in time until the "singularity" in the other generating function has been passed. This has the drawback of complicating the solution procedure, however.

Fortunately, with our approach, singularities in the generating functions are easily identified, as they are associated with singularities in the state transition matrix associated with the Hamiltonian system. Once the generating function $F_{2}$ is found, $\boldsymbol{p}$ and $\boldsymbol{x}_{o}$ can be derived from the necessary conditions associated with $F_{2}$ :

$$
\begin{aligned}
\boldsymbol{p} & =\frac{\partial F_{2}}{\partial \boldsymbol{x}}=F_{x x} \boldsymbol{x}+F_{x p_{o}} \boldsymbol{p}_{o} \\
\boldsymbol{x}_{o} & =\frac{\partial F_{2}}{\partial \boldsymbol{p}_{o}}=F_{p_{o} x} \boldsymbol{x}+F_{p_{o} p_{o}} \boldsymbol{p}_{o}
\end{aligned}
$$

Let us express $\boldsymbol{x}$ and $\boldsymbol{p}$ as a function of $\boldsymbol{x}_{o}$ and $\boldsymbol{p}_{o}$. From (49)

$$
\boldsymbol{x}=F_{p_{o} x}^{-1} \boldsymbol{x}_{o}-F_{p_{o} x}^{-1} F_{p_{o} p_{o}} \boldsymbol{p}_{o}
$$

Substituting this expression into (48),

$$
\boldsymbol{p}=F_{x x} F_{p_{o} x}^{-1} \boldsymbol{x}_{o}+\left(F_{x p_{o}}-F_{x x} F_{p_{o} x}^{-1} F_{p_{o} p_{o}}\right) \boldsymbol{p}_{o}
$$

These two equations can be combined into the matrix form:

$$
\left[\begin{array}{l}
\boldsymbol{x}(t) \\
\boldsymbol{p}(t)
\end{array}\right]=\left[\begin{array}{cc}
F_{p_{o} x}^{-1}\left(t, t_{0}\right) & -\left(F_{p_{o} x}^{-1} F_{p_{o} p 0}\right)\left(t, t_{0}\right) \\
\left(F_{x x} F_{p_{o} x}^{-1}\right)\left(t, t_{0}\right) & \left(F_{x p_{o}}-F_{x x} F_{p_{o} x}^{-1} F_{p_{o} p 0}\right)\left(t, t_{0}\right)
\end{array}\right]\left[\begin{array}{l}
\boldsymbol{x}\left(t_{0}\right) \\
\boldsymbol{p}\left(t_{0}\right)
\end{array}\right]
$$

Also if we define the state transition matrix as

$$
\Phi\left(t, t_{0}\right)=\left[\begin{array}{ll}
\phi_{x x}\left(t, t_{0}\right) & \phi_{x p}\left(t, t_{0}\right) \\
\phi_{p x}\left(t, t_{0}\right) & \phi_{p p}\left(t, t_{0}\right)
\end{array}\right]
$$

then the following expression holds:

$$
\left[\begin{array}{l}
\boldsymbol{x}(t) \\
\boldsymbol{p}(t)
\end{array}\right]=\left[\begin{array}{ll}
\phi_{x x}\left(t, t_{0}\right) & \phi_{x p}\left(t, t_{0}\right) \\
\phi_{p x}\left(t, t_{0}\right) & \phi_{p p}\left(t, t_{0}\right)
\end{array}\right]\left[\begin{array}{l}
\boldsymbol{x}\left(t_{0}\right) \\
\boldsymbol{p}\left(t_{0}\right)
\end{array}\right]
$$

From the fact that (52) and (54) should be equivalent, we can easily find the following relation between them:

$$
\begin{gathered}
F_{x x}=\phi_{p x} \phi_{x x}^{-1} \quad, \quad F_{x p_{o}}=\phi_{x x}^{-T} \\
F_{p_{o} x}=\phi_{x x}^{-1}, F_{p_{o} p_{o}}=-\phi_{x x}^{-1} \phi_{x p}
\end{gathered}
$$

These results indicate that $F_{2}$ is singular when $\phi_{x x}$ is singular. Also with the aid of Legendre transformation, it can be shown that $F_{1}$ is singular whenever $\phi_{x p}$ is singularg .

Consider the linear dynamics for our specific system, from which we can derive the state transition matrix:

$$
\left[\begin{array}{c}
\dot{\boldsymbol{x}} \\
\dot{\boldsymbol{p}}
\end{array}\right]=\left[\begin{array}{cc}
A & -B B^{T} \\
0 & -A^{T}
\end{array}\right]\left[\begin{array}{l}
\boldsymbol{x} \\
\boldsymbol{p}
\end{array}\right]=\bar{A}\left[\begin{array}{l}
\boldsymbol{x} \\
\boldsymbol{p}
\end{array}\right]
$$

\footnotetext{
${ }^{\mathrm{g}}$ Refer to Guibout and Scheeres ${ }^{13}$ for a more comprehensive analysis of singularities
} 
where

$$
\bar{A}=\left[\begin{array}{cc}
\bar{A}_{11} & \bar{A}_{12} \\
\bar{A}_{21} & \bar{A}_{22}
\end{array}\right]=\left[\begin{array}{l}
{\left[\begin{array}{llll}
0 & 0 & 1 & 0 \\
0 & 0 & 0 & 1 \\
3 & 0 & 0 & 2 \\
0 & 0 & -2 & 0
\end{array}\right]} \\
{\left[\begin{array}{llll}
0 & 0 & 0 & 0 \\
0 & 0 & 0 & 0 \\
0 & 0 & 0 & 0 \\
0 & 0 & 0 & 0
\end{array}\right] \quad\left[\begin{array}{cccc}
0 & 0 & 0 & 0 \\
0 & 0 & 0 & 0 \\
0 & 0 & -1 & 0 \\
0 & 0 & 0 & -1
\end{array}\right]} \\
\left.\begin{array}{cccc}
0 & 0 & -3 & 0 \\
0 & 0 & 0 & 0 \\
-1 & 0 & 0 & 0 \\
0 & -1 & -2 & 0
\end{array}\right]
\end{array}\right]
$$

Here we note that the $\bar{A}_{21}$ sub-matrix in $\bar{A}$ is always zero for the class of problems we consider here, namely problems where the "nominal" solution is no control $(\boldsymbol{p} \equiv 0$ for $\boldsymbol{x} \equiv 0)$. For expansions about an existing optimal control problem, the following observations are no longer true in general.

Now the state transition matrix is defined as

$$
\Phi\left(t, t_{0}\right)=e^{A\left(t-t_{0}\right)}
$$

whose explicit solution is given by

$$
\begin{aligned}
& \phi_{x x}=\left[\begin{array}{cccc}
4-3 \cos t & 0 & \sin t & -2 \cos t+2 \\
6 \sin t-6 t & 1 & -2+2 \cos t & -3 t+4 \sin t \\
3 \sin t & 0 & \cos t & 2 \sin t \\
6 \cos t-6 & 0 & -2 \sin t & -3+4 \cos t
\end{array}\right] \\
& \phi_{x p}=\left[\begin{array}{ccc}
-2.5 t \cos t+6.5 \sin t-4 t & -16 \cos t-5 t \sin t+16-3 t^{2} & \ldots \\
16 \cos t+5 t \sin t-16+3 t^{2} & -10 t \cos t+1.5 t^{3}+38 \sin t-28 t & \ldots \\
4 \cos t-4+2.5 t \sin t & -5 t \cos t+11 \sin t-6 t & \ldots \\
5 t \cos -11 \sin t+6 t & 28 \cos t+4.5 t^{2}+10 t \sin t-28 & \ldots \\
\ldots & -4 \cos t-2.5 t \sin t+4 & 5 t \cos t-11 \sin t+6 t \\
\ldots & -5 t \cos t+11 \sin t-6 t & -4.5 t^{2}+28-10 t \sin t-28 \cos t \\
\ldots & 1.5 \sin t-2.5 t \cos t & -6 \cos t+6-5 t \sin t \\
\ldots & 6 \cos t+5 t \sin t-6 & -9 t+18 \sin t-10 t \cos t
\end{array}\right] \\
& \phi_{p x}=0_{4 \times 4} \\
& \phi_{p p}=\left[\begin{array}{cccc}
4-3 \cos t & -6 \sin t+6 t & -3 \sin t & 6 \cos t-6 \\
0 & 1 & 0 & 0 \\
-\sin t & -2+2 \cos t & \cos t & 2 \sin t \\
2-2 \cos t & 3 t-4 \sin t & -2 \sin t & -3+4 \cos t
\end{array}\right]
\end{aligned}
$$

Computing the determinants symbolically, we find

$$
\begin{aligned}
\left|\phi_{x x}(t)\right| \equiv & \cos ^{2} t+\sin ^{2} t=1 \\
\left|\phi_{x p}(t)\right|= & 1536-30 t^{4} \cos t-250.5 t^{2}-2048 \cos t-912 t \sin t-18 t^{3} \sin 2 t \\
& +456 t \sin 2 t+\frac{27}{32} t^{4} \cos 2 t-139.5 t^{2} \cos 2 t+\frac{75}{16} t^{6}+512 \cos 2 t \\
& +400 t^{2} \cos t+222 t^{3} \sin t-\frac{1147}{32} t^{4}
\end{aligned}
$$

17 of 22 


$$
\begin{aligned}
\left|\phi_{p x}(t)\right| & \equiv 0 \\
\left|\phi_{p p}(t)\right| & \equiv\left(\cos ^{2} t+\sin ^{2} t\right)^{2}=1
\end{aligned}
$$

For optimal control problems of this class, the $\phi_{x x}$ matrix is the state transition matrix of the dynamical system, and for well-defined dynamical systems this matrix is never singular. We see this explicitly above. In fact, this should hold for all optimal control problems for which we expand the generating functions about a zero solution, as the $\bar{A}_{21}$ sub-matrix will always be zero and allow the $\phi_{x x}$ sub-matrix dynamics to decouple from the other sub-matrices. For the applications in [14], the corresponding matrix was only a sub-element of the state transition matrix, and hence could be singular without violating singularity of the entire state transition matrix (and indeed, was singular at certain times). Thus, we see that $F_{2}$ can never suffer this sort of singularity.

However, we are more interested in the occurrence of singularities of the matrix $\phi_{x p}$, as it affects whether $F_{1}$ becomes singular or not. (Note that it is $F_{1}$ which plays a key part in evaluating optimal feedback control and the optimal trajectory.) For that purpose, the time history of the determinant of $\phi_{x p}$ is shown in Figure 15. From this, for our particular system, it is clear that $\phi_{x p}$ is never singular except the initial epoch where the singularity is inherent, and thus that our optimal control is well defined and unique.

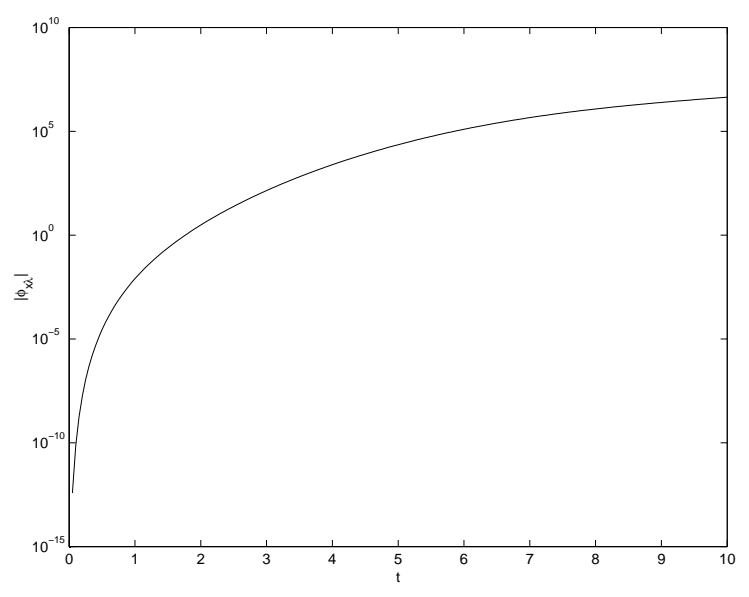

Figure 15. Determinant of $\phi_{x p}(t)$

\section{Conclusion}

We have proposed a new method of evaluating an optimal trajectory as well as an optimal feedback control via generating functions, which has been successfully applied to the continuous thrust optimal rendezvous problem relative to a circular orbit. In contrast to the prevalent results in the literature based on linearized dynamics, we considered the nonlinear system by performing a Taylor series expansion of the system dynamics, and showed that the introduction of higher order terms results in numerical convergence to the (unapproximated) nonlinear solution. Finally, we considered the possibility of singularities existing in our control procedure, and showed that they are absent in general for the particular application we are considering.

Our method has an advantage over the conventional numerical shooting method in the sense that it does not require that one should guess the initial or terminal adjoints. It also has an advantage over the method based on linearized dynamics in the sense that our higher order solution enhances the numerical precision and the region of convergence to the nonlinear reference solution. All these favorable results imply that our new method can be considered as an alternative and effective way of solving nonlinear optimal rendezvous problems. Furthermore, in addition to computing the optimal trajectory, our proposed optimal feedback control law can be used as an improved guidance law. 


\section{Appendix: Properties of Hamiltonian Systems and their Application to Boundary Value Problems}

\section{Hamiltonian System and Canonical Transformation}

This appendix briefly reviews Hamiltonian dynamical systems. See Greenwood ${ }^{19}$ for a more comprehensive discussion. Suppose we have a system whose equations of motion can be represented using Hamilton's canonical form

$$
\left[\begin{array}{c}
\dot{q}(t) \\
\dot{p}(t)
\end{array}\right]=\left[\begin{array}{r}
\frac{\partial H}{\partial p}(q(t), p(t), t) \\
-\frac{\partial H}{\partial q}(q(t), p(t), t)
\end{array}\right]
$$

where $H=H(q(t), p(t), t)$ is the Hamiltonian of the system, $q(t)=\left[\begin{array}{lll}q_{1}(t) & q_{2}(t) & \cdots\end{array} q_{n}(t)\right]^{T}$ is the generalized coordinate vector, and $p(t)=\left[\begin{array}{llll}p_{1}(t) & p_{2}(t) & \cdots & p_{n}(t)\end{array}\right]^{T}$ is the generalized momentum vector conjugate to $q(t)$. Suppose furthermore that there exists a canonical transformation from $(q, p)$ to a new set of coordinate $(Q, P)$ which is related by

$$
Q=Q(q, p, t), P=P(q, p, t)
$$

Then there exists a Hamiltonian $K=K(Q(t), P(t), t)$ in the new set of coordinates such that the equations of motion is of the form

$$
\left[\begin{array}{c}
\dot{Q} \\
\dot{P}
\end{array}\right]=\left[\begin{array}{r}
\frac{\partial K}{\partial P}(Q, P, t) \\
-\frac{\partial K}{\partial Q}(Q, P, t)
\end{array}\right]
$$

In order to relate $K$ with $H$, let us recall Hamilton's principle

$$
\delta I=\delta \int_{t_{0}}^{t_{f}} L d t=0
$$

where the Lagrangian $L$ is defined as $L(q, \dot{q}, t)=p^{T} \dot{q}-H(q, p, t)$. From (66) we have, both in old and new coordinates,

$$
\delta \int_{t_{0}}^{t_{f}}\left(p^{T} \dot{q}-H(q, p, t)\right) d t=\delta \int_{t_{0}}^{t_{f}}\left(P^{T} \dot{Q}-K(Q, P, t)\right) d t=0
$$

which implies that the integrands of the two integrals differ at most by a total time derivative of an arbitrary function $F$,i.e.,

$$
p^{T} \dot{q}-H(q, p, t)=P^{T} \dot{Q}-K(Q, P, t)+\frac{d F}{d t}
$$

Such a function is called a generating function and is a function of both old and new coordinates and time. However, from the $2 n$ relations (64) it turns out that $F$ is a function of $2 n+1$ variables instead of $4 n+1$ variables. Let us assume that $\mathrm{F}$ is dependent upon $n$ old coordinates and $n$ new coordinates. Then the generating function has one of the following forms ${ }^{19}$

$$
F_{1}\left(q, Q, t ; t_{0}\right), \quad F_{2}\left(q, P, t ; t_{0}\right), \quad F_{3}\left(p, Q, t ; t_{0}\right), \quad F_{4}\left(p, P, t ; t_{0}\right)
$$

If, for instance, $q$ and $Q$ are independent variables, then $F_{1}$ should be used. Expanding the total time derivative of $F_{1}$ yields

$$
\frac{d}{d t} F_{1}\left(q, Q, t ; t_{0}\right)=\frac{\partial F_{1}^{T}}{\partial q} \dot{q}+\frac{\partial F_{1}^{T}}{\partial Q} \dot{Q}+\frac{\partial F_{1}}{\partial t} .
$$


Substitution of (70) into (68) leads to

$$
\left(p-\frac{\partial F_{1}}{\partial q}\right)^{T} \dot{q}-H=\left(P+\frac{\partial F_{1}}{\partial Q}\right)^{T} \dot{Q}-K+\frac{\partial F_{1}}{\partial t}
$$

which is equivalent to

$$
\begin{aligned}
p & =\frac{\partial F_{1}}{\partial q}\left(q, Q, t ; t_{0}\right) \\
P & =-\frac{\partial F_{1}}{\partial Q}\left(q, Q, t ; t_{0}\right) \\
K(Q, P, t) & =H(q, p, t)+\frac{\partial F_{1}}{\partial t}\left(q, Q, t ; t_{0}\right)
\end{aligned}
$$

Similarly, if $q$ and $P$ are independent variables, then (68) can be rewritten as a function of two independent variables $q$ and $P$

$$
p^{T} \dot{q}-H(q, p, t)=-Q^{T} \dot{P}-K(Q, P, t)+\frac{d F_{2}}{d t}
$$

which yields

$$
\begin{aligned}
p & =\frac{\partial F_{2}}{\partial q}\left(q, P, t ; t_{0}\right) \\
Q & =\frac{\partial F_{2}}{\partial P}\left(q, P, t ; t_{0}\right) \\
K(Q, P, t) & =H(q, p, t)+\frac{\partial F_{2}}{\partial t}\left(q, P, t ; t_{0}\right) .
\end{aligned}
$$

Furthermore, it can be verified that the Legendre transformation

$$
F_{2}\left(q, P, t ; t_{0}\right)=F_{1}\left(q, Q, t ; t_{0}\right)+P^{T} Q
$$

relates $F_{1}$ with $F_{2}$. The same procedure leads to the similar results for $F_{3}\left(p, Q, t ; t_{0}\right)$ and $F_{4}\left(p, P, t ; t_{0}\right)$.

\section{Application to Boundary Value Problems}

Consider again the canonical transformation $(64)$ where $(q, p)$ and $(Q, P)$ satisfy the canonical equations of motion (63) and (65) subject to the Hamiltonian $H=H(q, p, t)$ and $K=K(Q, P, t)$, respectively. Here the variables $(Q, P)$ can be chosen to be constants by setting the new Hamiltonian $K \equiv 0$. That is

$$
\left[\begin{array}{c}
\dot{Q} \\
\dot{P}
\end{array}\right]=\left[\begin{array}{c}
\frac{\partial K}{\partial P} \\
-\frac{\partial K}{\partial Q}
\end{array}\right] \equiv 0 \Rightarrow\left[\begin{array}{c}
Q \\
P
\end{array}\right] \equiv \text { constant }
$$

And Eqs. (74) and (78) become

$$
\begin{aligned}
& \frac{\partial F_{1}}{\partial t}+H\left(q, \frac{\partial F_{1}}{\partial q}, t\right)=0 \\
& \frac{\partial F_{2}}{\partial t}+H\left(q, \frac{\partial F_{2}}{\partial q}, t\right)=0
\end{aligned}
$$

both of which are often referred to as the Hamilton-Jacobi $(\mathrm{HJ})$ equation. Indeed, they are equivalent; the only difference between the two is in their "initial boundary conditions". This difference, however, leads to a very different time evolution and even leads to the functions becoming singular at different epochs ${ }^{14}$.

20 of 22 
For an application to the boundary value problem, let us simply choose the initial conditions of the trajectory to be the constants of motion and solve the Hamilton-Jacobi equation for the generating function. In order to solve the Hamilton-Jacobi equation, the value of the generating function needs to be specified at some epoch. At $t=0$, both old and new coordinates are equal, therefore the generating function must define an identity transformation. $F_{1}$ cannot generate such a transformation since the initial and final positions are equal and not independent at $t=t_{0}$, thus $F_{1}$ is undefined at $t=t_{0}$. On the contrary, $F_{2}$ is well defined at $t=t_{0}$. In fact, if both $H$ and $F_{2}$ are analytic , then

$$
F_{2}\left(q, P, t=t_{0} ; t_{0}\right)=q^{T} P
$$

is the unique possible expression and defines the identity transformation at $t=t_{0}$. Therefore, given the Hamiltonian of a system we can solve the $\mathrm{HJ}$ equation for $F_{2}$ from the initial time. $F_{1}$ can only be solved if it is known at some other epoch than the initial time.

The main advantage of this approach is that once the generating function has been found, the unknown boundary conditions are simply evaluated from the algebraic manipulation of (72)- (73) and (76)- (77) without solving a differential equation.

\section{Acknowledgements}

The work described here was funded in part by the National Science Foundation by grant CMS 0408542 and by the Interplanetary Network Technology Program by a grant from the Jet Propulsion Laboratory, California Institute of Technology which is under contract with the National Aeronautics and Space Administration. 


\section{References}

1 Lawden, D. F., Optimal Trajectories for Space Navigation, Butterworths, London, England, 1963.

2 Billik, B. H., "Some Optimal Low-Acceleration Rendezvous Maneuvers," AIAA Journal, Vol. 2, No. 3, 1964, pp. 510-516.

3 London, H. S., "Second Approximation to the Solution of the Rendezvous Equations," AIAA Journal, Vol. 1, No. 7, 1963, pp. 1691-1693.

4 Anthony, M. L., and Sasaki, F. T., "Rendezvous Problem for Nearly Circular Orbits,", AIAA Journal, Vol. 3, No. 9, 1965, pp. 1066-1073.

5 Euler, E. A., “Optimal Low-Thrust Rendezvous Control,” AIAA Journal, Vol. 7, No. 6, 1969, pp. 1140-1144.

6 Jezewski, D. J., and Stoolz, J. M., "A Closed-Form Solution for Minimum-Fuel, Constant-Thrust Trajectories," AIAA Journal, Vol. 8, No. 7, 1970, pp. 1229-1234.

7 Marec, J., Optimal Space Trajectories, Elsevier, New York, NY, 1979.

8 Carter, T. E., "Fuel-Optimal Maneuvers of a Spacecraft Relative to a Point in Circular Orbit," Journal of Guidance, Control, and Dynamics, Vol. 7, No. 6, 1984, pp. 710-716.

9 Carter, T., and Humi, M., "Fuel-Optimal Rendezvous Near a Point in General Keplerian Orbit," it Journal of Guidance, Control, and Dynamics, Vol. 10, No. 6, 1987, pp. 567-573.

${ }^{10}$ Humi, M., "Fuel-Optimal Rendezvous in a General Central Gravity Field," it Journal of Guidance, Control, and Dynamics, Vol. 16, No. 1, 1993, pp. 215-217.

11 Carter, T. E., and Pardis, C. J., "Optimal Power-Limited Rendezvous with Upper and Lower Bounds on Thrust," it Journal of Guidance, Control, and Dynamics, Vol. 19, No. 5, 1996, pp. 1124-1133.

12 Lembeck, C. A., and Prussing, J. E., "Optimal Impulsive Intercept with Low-Thrust Rendezvous Return," it Journal of Guidance, Control, and Dynamics, Vol. 16, No. 3, 1993, pp. 426-433.

13 Guibout, V., and Scheeres, D. J., "Solving Relative Two Point Boundary Value Problems: Applications to Spacecraft Formation Flight Transfers," Journal of Guidance, Control, and Dynamics, Vol. 27, No. 4, 2004, pp. 693-704.

14 Guibout, V., and Scheeres, D. J., "Solving Two Point Boundary Value Problems using Generating Functions: Theory and Applications to Astrodynamics," Astrodynamics Book Series, (submitted for publication), Elsevier.

15 Park, C., and Scheeres, D. J., "Solutions of Optimal Feedback Control Problem using Hamiltonian Dynamics and Generating Functions," Proceedings of the 42nd IEEE Conference on Decision and Control, IEEE, Maui, Hawaii, Dec 9-12, 2003, pp. 1222-1227.

16 Scheeres, D. J., Park, C., and Guibout, V. M., "Solving Optimal Control Problems with Generating Functions," Proceedings of the AAS/AIAA Astrodynamics Specialist Conference, AAS/AIAA, Big Sky, Montana, Aug 3-7, 2003, Paper AAS 03-575.

17 Park, C., and Scheeres, D. J., "A Generating Function for Optimal Feedback Control Laws that Satisfies the General Boundary Conditions of a System," Proceedings of the 23rd American Control Conference, AACC, Boston, MA, June 30-July 2, 2004, pp. 679-684.

18 Park, C., and Scheeres, D. J., "Solving Optimal Feedback Control Problem with General Boundary Conditions using Hamiltonian Dynamics and Generating Functions," Automatica, (submitted for publication).

19 Greenwood, D. T., Classical Dynamics, Prentice-Hall, Englewood Cliffs, NJ, 1977, pp.187-271.

20 Bryson, A. E., and Ho, Y., Applied Optimal Control, Taylor \& Francis, Briston, PA, 1975, pp. 148176. 